\title{
Modulation of Olfactory Perception by Visual Cortex Stimulation
}

\author{
Jahan B. Jadauji, ${ }^{1}$ Jelena Djordjevic, ${ }^{1}$ Johan N. Lundström, ${ }^{2,3,4}$ and Christopher C. Pack ${ }^{1}$ \\ ${ }^{1}$ Montreal Neurological Institute, McGill University, Montreal, Quebec H3A 2B4, Canada, ${ }^{2}$ Monell Chemical Senses Center, Philadelphia, Pennsylvania \\ 19104, ${ }^{3}$ Department of Psychology, University of Pennsylvania 19104, Philadelphia, Pennsylvania, and ${ }^{4}$ Department of Clinical Neuroscience, Karolinska \\ Institute, SE-171 77 Stockholm, Sweden
}

When attempting to identify an object based on smell alone, people often visualize the perceived source of the odorant. This close association between olfactory and visual functions is supported by neuroimaging studies demonstrating activation of visual cortex during performance of purely olfactory tasks. Such activation might simply reflect the correlation between olfactory percepts and the corresponding visual images, or it might reflect a causal contribution of visual processing to olfactory perception. Here we provide evidence in support of the latter possibility. Using repetitive transcranial magnetic stimulation, we show that stimulating human visual cortex improves performance on a task requiring discrimination among different odor qualities. No significant improvement is found for tasks involving discrimination between intensities of the same odor, from stimulation of auditory cortex, or from "sham" stimulation. These results are thus consistent with a specific visual cortical influence on high-level olfactory perception. They also demonstrate that unimodal perceptual tasks are influenced by processing within cortical areas of other, seemingly unrelated, sensory systems.

\section{Introduction}

When presented with the smell of a strawberry, people often form a mental image of a strawberry. Analogously, neuroimaging studies have reported activation of visual cortical areas during the performance of various purely olfactory tasks (Royet et al., 1999; Qureshy et al., 2000; Zatorre et al., 2000; Dade et al., 2002). One possible explanation for these visual-olfactory interactions is that subjects decide upon the identity of the stimulus, based entirely on processing within olfactory brain regions, after which they visualize the corresponding object. In this case, visual cortical activity would be correlated with olfactory perception, but it would have no influence over the percept.

Alternatively, the visual cortex activation might reflect a process by which activity in visual brain regions is incorporated into the processing of the olfactory stimulus. For instance the process of attempting to visualize the object associated with an olfactory stimulus might improve the ability to identify that object. In that case, experimental manipulations that influence visual cortex activity would be expected to influence olfactory perception. Distinguishing between these two possibilities is important for understanding the nature of cross-modal sensory processing: Does activation of brain regions that are primarily dedicated to

Received Dec. 4, 2011; revised Jan. 10, 2012; accepted Jan. 18, 2012.

Author contributions: J.B.J., J.D., J.N.L., and C.C.P. designed research; J.B.J. performed research; J.B.J., J.D., J.N.L., and C.C.P. analyzed data; J.B.J., J.D., J.N.L., and C.C.P. wrote the paper.

This work was supported by grants from the Centre of Excellence in Commercialization and Research and the Alfred P. Sloan Foundation to C.C.P., as well as the Swedish Research Council (2009-2337) to J.N.L., and an Natural Sciences and Engineering Research Council of Canada Discovery grant (355938-08) to J.D. We thank Drs. Olivier Collignon and Robert Zatorre for helpful comments on the manuscript. We thank Anna Engel for additional support.

Correspondence should be addressed to Christopher C. Pack, Montreal Neurological Institute, McGill University, 3801 University St., Montreal, QC, Canada. E-mail: christopher.pack@mcgill.ca.

DOI:10.15 23/JNEUROSCI.6022-11.2012

Copyright $\odot 2012$ the authors $\quad 0270-6474 / 12 / 323095-06 \$ 15.00 / 0$ one sensory modality influence processing in other sensory modalities?

One method of manipulating cortical activity is to use transcranial magnetic stimulation (TMS) to introduce a small electrical current in a targeted brain region. Previous work has shown that repetitive application of TMS (rTMS) can affect brain activity and behavioral performance for time periods lasting from several minutes to several hours (Huang et al., 2005; Tegenthoff et al., 2005; Waterston and Pack, 2010). With respect to vision, rTMS has been shown to improve performance on perceptual tasks (Thompson et al., 2008; Waterston and Pack, 2010) and to exert long-lasting effects on neuronal firing and synchrony (Pasley et al., 2009). Thus rTMS provides an ideal method for testing the hypothesis that visual cortical activation influences olfactory perception.

Here we show that rTMS activation of early visual cortex improves performance on a task that requires discrimination among different odorants. No improvement is found following stimulation of primary auditory cortex or for tasks that require simply detecting the intensity of an odorant. These results are thus consistent with a specific influence of visual cortical activation on a high-level olfactory task. More generally, these results show that activation of an early sensory area can shape performance on a relevant task in a different sensory modality.

\section{Materials and Methods}

Experiment $1-V$-TMS

Participants. Twenty individuals (10 female; mean age 24) participated in Experiment 1 involving visual and olfactory testing. All subjects were naive as to the aims of the study and also as to which TMS coil was used in a given session. Exclusion criteria included metal implants, prostheses, family history of epilepsy or other neurological disorder, pregnancy, and use of antidepressant medications. All subjects provided written and 
informed consent, and all aspects of the recruitment procedures and experimental protocols were approved by the Ethics Review Board of the Montreal Neurological Institute.

rTMS stimulation. Sessions involving TMS were performed using either a real TMS or a "sham" TMS coil. For real TMS stimulation over visual cortex (V-TMS), a Magstim Rapid 2 Air Film stimulating coil was used. Sham stimulation (sham-TMS) used the Magstim Air Film Placebo coil to replicate the feeling of TMS while delivering only a very weak current to the brain $(<0.3 \mathrm{~T})$.

Real TMS was delivered via a continuous theta burst protocol, using the parameters described by Huang et al. (2005). Stimulation consisted of 200 bursts of three pulses delivered at $50 \mathrm{~Hz}$, and a burst frequency of 5 $\mathrm{Hz}$ for a total of 600 pulses delivered over the course of the $41 \mathrm{~s}$ stimulation. This protocol has several advantages over both high and lowfrequency continuous stimulation (Huang et al., 2005). First, it provides longer-lasting effects, which were necessary for olfactory testing; second, it appears to provide more stable and reliable effects, as estimated from intersubject variability; third, it requires relatively little time to apply ( $\sim 40 \mathrm{~s}$ ), which reduces any discomfort experienced by subjects. Consistent with these findings, our previous work has shown that theta burst rTMS influences visual perception both more quickly and more reliably than low-frequency (1 Hz) stimulation (Waterston and Pack, 2010). Because of this latter result, we have used continuous theta burst here, despite the fact that it was found in previous work to exert an inhibitory influence on motor thresholds (Huang et al., 2005). The same study showed a facilitatory effect of intermediate theta burst, but we have not explored this protocol in the domain of visual perception.

For the regular TMS session, the coil was placed over V1 using the inion as a starting point and then using phosphene detection in both hemispheres. Similarly, the sham coil was placed using the inion as a reference point. In both cases the coil was placed gently but securely against the subject's head; the coil housing was oriented so that it was flat against the skull with the center point over the desired location. As the connectivity between visual cortex and olfactory brain regions is not well understood, we stimulated both hemispheres to maximize the possibility of observing cross-modal interactions.

Visual stimuli and procedure. Subjects viewed the stimuli at a distance of $57 \mathrm{~cm}$ from the display. Stimuli were generated using the Psychophysics toolbox (Brainard, 1997; Pelli, 1997) and drawn on a HPA2717A CRT monitor at a refresh rate of $75 \mathrm{~Hz}$. Responses were collected using a computer game pad with Matlab (MathWorks).

Subjects pressed a button on a keyboard to start each trial. The visual stimulus was a Gabor patch $\left(1.5^{\circ}\right.$ radius, 0.75 cycles/degree, $27 \mathrm{~ms}$ duration) presented at an eccentricity of $6^{\circ}$ to the right of a central fixation point (Fig. 1A). This location was chosen based on the eccentricity of phosphenes in pilot studies and previous work using a similar setup (Waterston and Pack, 2010). Subjects performed a two-alternative, forced-choice task by indicating after each stimulus presentation whether the Gabor patch was oriented vertically or horizontally (Fig. $1 B)$. To modulate task difficulty, we varied stimulus contrast, after determining each subject's contrast threshold in preliminary testing. Contrast thresholds were obtained via a staircase procedure in which contrast was decreased in increments of $0.1 \%$ after three correct trials and increased by the same amount following one incorrect trial until a criterion level of $\sim 75 \%$ accuracy was reached. Subjects then performed 200 visual trials at this fixed contrast. Subjects then received either no-TMS, the sham-TMS or V-TMS, depending on the session. Next, subjects performed 200 more visual trials at the same fixed contrast. In the no-TMS session, a brief pause of approximately the same duration as it took to administer rTMS was introduced to mimic the other sessions. In addition, subjects received a $30 \mathrm{~s}$ break after each 40 trials to avoid eye strain.

Odor stimuli and procedure. Olfactory stimuli were delivered via the Sniffin' Sticks odor delivery kit (Hummel et al., 1997), which consists of marker-like capped containers in which odorants at various concentrations can be placed (Burghart MedizinTechnik). Subjects were blindfolded for all olfactory testing.

Each olfactory testing session began with olfactory thresholding (described below), after which we applied either real TMS, sham TMS or no TMS. Subjects then performed the intensity discrimination task, which was followed by the quality discrimination task (Fig. 1C). Both tasks are described in detail below.

Odor quality discrimination task. Subjects were presented with three iso-intense odors in random order, two being of identical quality and one being of a different quality; the task was to identify which of the three was of a different quality (described in detail by Lundström et al., 2008). The odors in this task were all easily detectable and presented with an interstimulus interval of $30 \mathrm{~s}$ between each triplet, using 16 repetitions of each unique combination.

Perithreshold odor intensity discrimination task. As described in detail previously (Lundström et al., 2008), we determined each subject's olfactory detection threshold for the odorant $n$-butanol, using a threealternative, forced-choice, seven reversals, staircase paradigm; the mean of the last four reversals was used to estimate detection threshold. This detection threshold value was subsequently used to set the baseline for the perithreshold intensity discrimination test.

Following the threshold measurements, subjects completed trials in which they were presented sequentially with two Sniffin' Sticks containing the odorant $n$-butanol. One stimulus was always at the subject's detection threshold, and the other was one concentration step above or below the threshold. Subjects were then asked to indicate which odor was stronger. Each stimulus was presented for $\sim 3 \mathrm{~s}$ with the second following directly after the first. Each trial was separated by $\sim 20 \mathrm{~s}$ with a total of 20 repetitions in each session.

Sessions. In Experiment 1, subjects participated in six primary sessions, three for visual testing and three for olfactory testing (Fig. 1D). The three conditions for each modality were: (1) V-TMS stimulation, (2) shamTMS stimulation, and (3) a no-TMS control. Sessions were held with at least one full day between them to eliminate any potential residual rTMS effects. The first three sessions were devoted to visual testing, with the first session involving a no-TMS control to familiarize the subject with the visual discrimination task and to record a no-TMS baseline. The two 
subsequent sessions involved real or sham TMS, with the order alternating from one subject to the next.

The same sequence of sessions was used for olfactory testing (sessions 4-6). In each session, we initially determined the subject's olfactory threshold as described above, after which the subject received no-TMS, regular TMS over V1 (left and right) or the sham-TMS over V1 (left and right). This was followed by administration of the intensity discrimination task, a brief pause, and then the quality discrimination task. To avoid practice effects and odor habituation, we did not administer the olfactory discrimination tasks before the application of TMS. Thus, in the analysis described above, the baseline for olfactory performance was taken from the no-TMS session.

\section{Experiment 2-auditory TMS}

Fourteen of the original 20 subjects participated in Experiment 2, which involved stimulation of auditory cortex (A1). Because of difficulties associated with retaining subjects over multiple sessions, no A1 sham sessions were performed. As with V-TMS sessions, we first determined subjects' olfactory thresholds before application of rTMS. Positioning of the TMS coil over auditory cortex was accomplished using a procedure described previously (Langguth et al., 2006). Briefly, the coil was placed at the $\mathrm{T} 3$ position, and then moved $\sim 2.5 \mathrm{~cm}$ upward along the T3-Cz line and $\sim 1.5 \mathrm{~cm}$ posterior, perpendicular to the T3-Cz line. The center of the coil was just above the ear in the majority of subjects. Upon completion of rTMS, subjects performed the olfactory tasks in the same manner and in the same order as described above for Experiment 1.

\section{Results}

To examine the possibility that visual cortex activation influences olfactory perception, we tested subjects' olfactory perception before and after application of rTMS directed toward the visual cortex, using an rTMS protocol that has previously been shown to improve visual processing (Waterston and Pack, 2010). Because rTMS modulates brain activity in the targeted area, it provides a powerful test of the hypothesis that visual cortex activation modulates olfactory perception.

\section{Influence of visual cortex rTMS on visual perception}

To verify that rTMS modulated cortical processing, we first determined how visual cortex rTMS affected visual perception. Specifically we asked subjects to indicate the orientation of a briefly presented Gabor pattern (Fig. 1A). For each subject, stimulus contrast was adjusted during preliminary thresholding so that performance was $\sim 75 \%$ correct. Subjects then performed 200 trials at threshold contrast before and after the application of bilateral theta burst stimulation (Waterston and Pack, 2010) (the V-TMS condition). In a separate session, subjects took part in control experiments involving "sham" TMS, which replicated the feeling of rTMS stimulation without the application of any current. Additionally, as a baseline measure, we tested subjects on a condition in which no TMS was applied.

Figure 2 shows the results of these visual testing sessions for all 20 subjects. Each point corresponds to the percentage of correct responses for one subject before and after the application of rTMS (or in consecutive blocks for the no-TMS condition). Across subjects, the mean change in visual discrimination performance was $0.1 \%$ ( $\mathrm{SD} \pm 5.3$ ) for the no-TMS condition, $2.3 \%$ ( $\mathrm{SD} \pm 4.9$ ) for the sham-TMS condition, and 5.5\% (SD \pm 5.7 ) for the $\mathrm{V}$-TMS condition.

A repeated-measures ANOVA revealed a significant difference in visual discrimination performance across sessions $\left(F_{(2,19)}=5.57\right.$, $p=0.017$ ), and subsequent linear Scheffe's post hoc tests yielded a significant difference between the V-TMS and the no-TMS conditions $(p=0.012)$, as well as between the V-TMS and the shamTMS conditions $(p=0.037)$. No significant difference was found
A
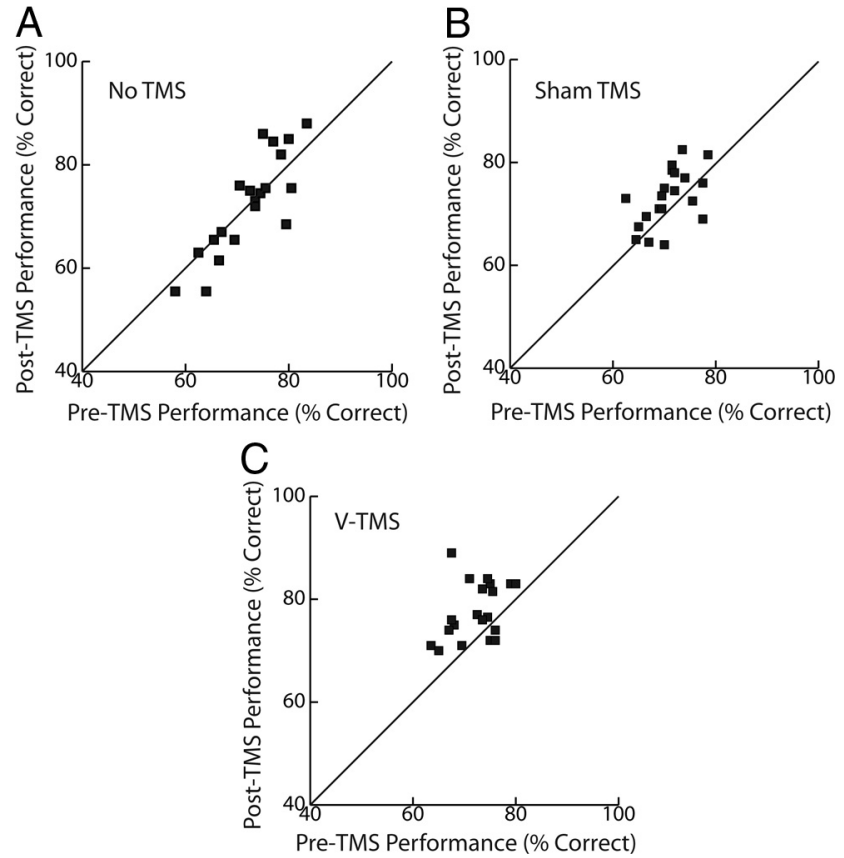

Figure 2. Performance on the visual task for the No TMS (A), Sham TMS (B) , and V-TMS ( $\boldsymbol{C}$ sessions. Plots show pre-TMS performance against post-TMS performance. Average improvements for following the TMS condition are $0.1 \%, 2.3 \%$, and $5.5 \%$ for No TMS, Sham TMS, and V-TMS.

between the no-TMS and sham-TMS conditions $(p=0.301)$. Importantly, only the change in percentage correct following real TMS was significantly different from zero, as assessed with separate one-sample Student's $t$ tests $\left(t_{(19)}=4.28, p<0.001\right)$. To account for any improvements due to practice effects, we used the change in percentage correct from the no-TMS session as a baseline measure of improvement within a session; this value was subsequently subtracted from those obtained in the sham- and $\mathrm{V}$-TMS sessions. Only the V-TMS minus no-TMS change in percentage correct was significantly different from zero (Fig. 3), as demonstrated with a one-sample Student's $t$ test $\left(t_{(19)}=2.79, p=\right.$ 0.012).

We also verified that performance was stable within each session by comparing performance on the first and second 100 psychophysical trials with a paired-samples Student's $t$ test. We found no difference between these two blocks of trials $\left(t_{(19)}=\right.$ $0.519, p>0.608)$, indicating that the improved perceptual performance began rapidly after stimulation and lasted for many minutes thereafter (Huang et al., 2005; Waterston and Pack, 2010).

\section{Influence of visual cortex rTMS on olfactory quality discrimination}

The previous results demonstrate that our rTMS protocol modulates visual cortical activity. The second phase of the experiment involved testing whether the same stimulation could modulate olfactory perception. To examine this possibility, we tested olfactory perception in the same 20 subjects under conditions that were analogous to those used in the visual testing sessions. Subjects participated in three sessions (no-TMS, sham-TMS and V-TMS) in which they were probed on an olfactory perithreshold intensity discrimination task and an olfactory quality discrimination task. The intensity discrimination task requires subjects to identify which of two stimuli has the stronger concentration of a 
single target odor ( $n$-butanol). This task is thought to probe absolute stimulus sensitivity by requiring subjects to assess the strength of an odorant based on instantaneously available information. On the other hand the quality discrimination task requires subjects to discriminate the odd odor from a group of three stimuli. Consequently it is considered a higher order olfactory task, as it demands the formation of a quality representation and its maintenance in working memory. Thus, while both tasks probe discrimination performance, we reasoned that the quality discrimination task might be more susceptible to the cognitive processes associated with multimodal integration.

We first analyzed our results with a repeated-measures ANOVA on the absolute percentage correct across the three TMS conditions; this demonstrated a significant effect of session $\left(F_{(2,19)}=3.78\right.$, $p<0.033)$. Pairwise analysis of the olfactory discrimination task yielded a significant difference only between performance during the V-TMS and no-TMS conditions $(p<0.035)$, with a statistical tendency toward an effect on performance between the V-TMS and sham-TMS sessions $(p=0.088)$ (Fig. 3 ). As in the visual task, we sought to eliminate practice effects by subtracting the change in performance during the no-TMS from the results of the V-TMS and sham-TMS sessions. rTMS over the visual cortex increased the ability of subjects to discriminate between odor qualities by an average of $10.92 \%$ (SD \pm 21.37 ), compared with a $3.98 \%$ (SD \pm 14.61 ) increase for the sham-TMS condition. Separate paired-sample Student's $t$ tests demonstrated that the improvement in odor quality discrimination following rTMS was significantly different from no-TMS $\left(t_{(19)}=2.28, p=0.034\right)$, whereas the performance increase following sham-TMS was not significantly different from no-TMS $\left(t_{(19)}=1.22, p=0.239\right)$.

\section{Influence of visual cortex rTMS on perithreshold odor intensity discrimination}

In contrast, the intensity discrimination task yielded no significant difference in performance across the three TMS sessions, as demonstrated by a repeated-measures ANOVA $\left(F_{(2,19)}=1.29\right.$, $p=0.288)$. Performance on the intensity discrimination task changed by an average of $2.5 \%$ ( SD \pm 16.4 ) following V-TMS and $-3.5 \%$ ( $\mathrm{SD} \pm 15.7$ ) following sham-TMS relative to the no-TMS condition. These baseline corrected values were not significantly different from zero (Student's $t$ tests, $t_{(19)}=0.681, p=0.504$ and $t_{(19)}=-0.995, p=0.332$ respectively; Fig. 3 ). Thus, rTMS of visual cortex improved olfactory perception in a task-specific manner.

\section{Influence of auditory cortex rTMS on olfactory perception}

To assess the specificity of the effect of V-TMS on olfactory discrimination, we repeated both the quality and intensity discrimination tasks with 14 of the subjects in a condition in which stimulation was directed toward primary auditory cortex (A1) (Langguth et al., 2006). The results of this auditory TMS (A-TMS) protocol are shown in Figure 3. As with the V-TMS condition, A1 stimulation had little effect on performance on the olfactory intensity discrimination task, as shown by a Student's one sample $t$ test (mean change $-0.71 \%$; $t_{(13)}=$
$-0.172, p=0.866$, Fig. 3). Similarly, no improvement was found following A-TMS on the olfactory discrimination task, and in fact, performance appeared to decrease (mean change $-9.82 \%)$, although this change was not significant $\left(t_{(13)}=\right.$ $-1.501, p=0.157)$. Thus the effects of rTMS appear to be specific to the site of the stimulation. Consistent with this idea, a repeated-measures ANOVA of the baseline corrected data indicated a significant difference on the quality discrimination task between the V-TMS and A-TMS conditions $\left(F_{(1,13)}=\right.$ 6.12, $p=0.028)$.

\section{Discussion}

We have shown that rTMS of early visual cortex influences olfactory perception. Specifically, we found a post-rTMS improvement in performance on a task requiring subjects to discriminate between odor qualities, and not on one requiring comparison of different intensities of the same odor. Sham rTMS did not yield any consistent improvements on either olfactory task. Similarly, there was no improvement on either olfactory task following rTMS of primary auditory cortex. These results are thus consistent with the idea that visual cortex activity exerts a specific influence on olfactory perception. More generally they provide a causal demonstration of the notion that visual representations are important for the formation of an odor quality percept.

\section{Comparison to prior work}

Several groups have previously reported that rTMS of visual cortex can improve performance on visual perceptual tasks (Thompson et al., 2008; Waterston and Pack, 2010; Tadin et al., 2011). In these studies, as in the current work, the effects of TMS were not specific to any particular stimulus, but rather, they appear to modulate cortical activity that was already present in response to the specific stimulus shown on a given trial. Mechanistically, rTMS might increase the amplitude of an existing visual representation, perhaps through disinhibition (Tadin et al., 2011), or it might reduce noise that impairs stimulus discrimination (Waterston and Pack, 2010). Either of these mechanisms could improve the fidelity of visual representations, and in the process contribute to cross-modal interactions that rely on vision.

Previous studies of the relationship between vision and olfaction have demonstrated that an odorant can draw visual attention to the semantically correspondent object in a complex visual scene (Seigneuric et al., 2010; Seo et al., 2010a,b) 
and that visual objects can modulate the neural processing of odors (Gottfried and Dolan, 2003; Seo et al., 2010a,b). Similarly, visual perception in a binocular rivalry task is dominated by the image that is associated with a simultaneously presented odorant of the same semantic quality (Zhou et al., 2010). In these experiments the semantic identity of the stimulus was crucial to the visual-olfactory interaction, supporting the notion that quality discrimination is a higher-order olfactory task.

There has been little previous work on rTMS and cross-modal interactions. In blind subjects visual cortex TMS interferes with both Braille reading and general tactile perception (Zangaladze et al., 1999; Kupers et al., 2007). However, it is difficult to compare studies using blind subjects to those using sighted subjects, as visual cortex in blind subjects is often sensitive to inputs from other modalities (Ptito et al., 2008).

\section{Possible anatomical basis of visual-olfactory crossmodal interaction}

Recently it has been suggested that, whereas the piriform cortex processes the initial stage of the formation of an odor quality percept (Lundström et al., 2011), the orbitofrontal cortex (OFC) is responsible for the final quality decision. Of particular relevance to the present work is a neuroimaging study (Savic et al., 2000) demonstrating that the OFC was activated during an odor quality discrimination task and not during an odor intensity discrimination task. Similarly, subjects with damage to the OFC are impaired at olfactory discrimination, but have normal olfactory detection (Zatorre and Jones-Gotman, 1991). These results are consistent with the general pattern of rTMS results reported here (Fig. 3), suggesting OFC as a possible substrate for integration of visual and olfactory stimuli (Rolls et al., 1996). Given the seemingly longlasting effects, this idea could be investigated by means of neuroimaging techniques to probe OFC activity before and after rTMS of the visual cortex.

Exactly how the visual cortex might influence processing in OFC is unknown. At present no monosynaptic connections between these regions are known to exist, and to date, few neuroimaging studies have explored the interaction of visual and higher-order odor perception. Thus the "feedforward" anatomical route that connects the visual cortex to higher-order olfactory perception is unknown, and indeed, our results are equally consistent with a "feedback" modulation of information from OFC to visual cortex.

\section{Limitations of the present study}

One possible explanation for our results is that rTMS of occipital cortex inhibits visual cortex activity, freeing up attentional resources so that the subject can focus on olfactory perception. Indeed, there is some evidence that our rTMS protocol is inhibitory (Huang et al., 2005), and competition across modalities is a well known phenomenon (Tellinghuisen and Nowak, 2003; Lavie, 2005). We consider this account to be unlikely for two reasons. First, the same rTMS protocol applied to auditory cortex did not improve olfactory perception (Fig. 3), indicating that generalized intermodality competition is unlikely to provide an explanation for our findings. Second, we blindfolded our subjects during performance of the olfactory tasks, so that there was little external visual input in any olfactory condition.

We have interpreted our results as evidence that that V-TMS has an effect on higher order olfaction. However, an alternative interpretation is that V-TMS might improve performance on higher order processing in general. Indeed, if V-TMS influences processing in OFC, one might expect modulation of performance in other domains in which OFC has been implicated, such as gustatory or somatosensory tasks (Rolls et al., 1996). More generally it is conceivable that V-TMS might even influence other cognitive tasks, such as language comprehension. Finally it is possible that cross-modal interactions could be evoked following purely sensory stimulation, rather than TMS, as recent work has shown long-lasting perceptual changes following high-frequency visual stimulation (Beste et al., 2011). These interesting possibilities await further testing.

\section{References}

Beste C, Wascher E, Güntürkün O, Dinse HR (2011) Improvement and impairment of visually guided behavior through LTP- and LTD-like exposure-based visual learning. Curr Biol 21:876-882.

Brainard DH (1997) The Psychophysics toolbox. Spat Vis 10:433-436.

Dade LA, Zatorre RJ, Jones-Gotman M (2002) Olfactory learning: convergent findings from lesion and brain imaging studies in humans. Brain 125:86-101.

Gottfried JA, Dolan RJ (2003) The nose smells what the eye sees: crossmodal visual facilitation of human olfactory perception. Neuron 39:375-386.

Huang YZ, Edwards MJ, Rounis E, Bhatia KP, Rothwell JC (2005) Theta burst stimulation of the human motor cortex. Neuron 45:201-206.

Hummel T, Sekinger B, Wolf SR, Pauli E, Kobal G (1997) 'Sniffin' sticks': olfactory performance assessed by the combined testing of odor identification, odor discrimination and olfactory threshold. Chem Senses 22:39-52.

Kupers R, Pappens M, de Noordhout AM, Schoenen J, Ptito M, Fumal A (2007) rTMS of the occipital cortex abolishes Braille reading and repetition priming in blind subjects. Neurology 68:691-693.

Langguth B, Zowe M, Landgrebe M, Sand P, Kleinjung T, Binder H, Hajak G, Eichhammer P (2006) Transcranial magnetic stimulation for the treatment of tinnitus: a new coil positioning method and first results. Brain Topogr 18:241-247.

Lavie N (2005) Distracted and confused?: selective attention under load. Trends Cogn Sci 9:75-82.

Lundström JN, Boesveldt S, Albrecht J (2011) Central processing of the chemical senses: an overview. ACS Chem Neurosci 2:5-16.

Lundström JN, Boyle JA, Jones-Gotman M (2008) Body positiondependent shift in odor percept present only for perithreshold odors. Chem Senses 33:23-33.

Pasley BN, Allen EA, Freeman RD (2009) State-dependent variability of neuronal responses to transcranial magnetic stimulation of the visual cortex. Neuron 62:291-303.

Pelli DG (1997) The VideoToolbox software for visual psychophysics: transforming numbers into movies. Spat Vis 10:437-442.

Ptito M, Fumal A, de Noordhout AM, Schoenen J, Gjedde A, Kupers R (2008) TMS of the occipital cortex induces tactile sensations in the fingers of blind Braille readers. Exp Brain Res 184:193-200.

Qureshy A, Kawashima R, Imran MB, Sugiura M, Goto R, Okada K, Inoue K, Itoh M, Schormann T, Zilles K, Fukuda H (2000) Functional mapping of human brain in olfactory processing: a PET study. J Neurophysiol $84: 1656-1666$.

Rolls ET, Everitt BJ, Roberts A (1996) The orbitofrontal cortex. Philos Trans R Soc Lond Ser B Biol Sci 351:1433-1444.

Royet JP, Koenig O, Gregoire MC, Cinotti L, Lavenne F, Le Bars D, Costes N, Vigouroux M, Farget V, Sicard G, Holley A, Mauguière F, Comar D, Froment JC (1999) Functional anatomy of perceptual and semantic processing for odors. J Cogn Neurosci 11:94-109.

Savic I, Gulyas B, Larsson M, Roland P (2000) Olfactory functions are mediated by parallel and hierarchical processing. Neuron 26:735-745.

Seigneuric A, Durand K, Jiang T, Baudouin JY, Schaal B (2010) The nose tells it to the eyes: crossmodal associations between olfaction and vision. Perception 39:1541-1554.

Seo HS, Arshamian A, Schemmer K, Scheer I, Sander T, Ritter G, Hummel T 
(2010a) Cross-modal integration between odors and abstract symbols. Neurosci Lett 478:175-178.

Seo HS, Roidl E, Müller F, Negoias S (2010b) Odors enhance visual attention to congruent objects. Appetite 54:544-549.

Tadin D, Silvanto J, Pascual-Leone A, Battelli L (2011) Improved motion perception and impaired spatial suppression following disruption of cortical area MT/V5. J Neurosci 31:1279-1283.

Tegenthoff M, Ragert P, Pleger B, Schwenkreis P, Förster AF, Nicolas V, Dinse HR (2005) Improvement of tactile discrimination performance and enlargement of cortical somatosensory maps after $5 \mathrm{~Hz}$ rTMS. PLoS Biol 3:e362.

Tellinghuisen D, Nowak E (2003) The inability to ignore auditory distractors as a function of visual task perceptual load. Percept Psychophys 65:817-828.

Thompson B, Mansouri B, Koski L, Hess RF (2008) Brain plasticity in the adult: modulation of function in amblyopia with rTMS. Curr Biol 18:1067-1071.

Waterston ML, Pack CC (2010) Improved discrimination of visual stimuli following repetitive transcranial magnetic stimulation. PLoS One 5:e10354.

Zangaladze A, Epstein CM, Grafton ST, Sathian K (1999) Involvement of visual cortex in tactile discrimination of orientation. Nature 401:587-590.

Zatorre RJ, Jones-Gotman M (1991) Human olfactory discrimination after unilateral frontal or temporal lobectomy. Brain 114:71-84.

Zatorre RJ, Jones-Gotman M, Rouby C (2000) Neural mechanisms involved in odor pleasantness and intensity judgments. Neuroreport 11:2711-2716.

Zhou W, Jiang Y, He S, Chen D (2010) Olfaction modulates visual perception in binocular rivalry. Curr Biol 20:1356-1358. 